\title{
Design of Manipulator PLC Control System
}

\author{
Yan Bao \\ Research Department, Jilin Teacher's Institute of Engineering and Technology, Changchun, Jilin, \\ china 130052 \\ zxr67811@163.com
}

\begin{abstract}
Keywords: CNC lathe; Automatic programming; Processing technology; Parameter optimization; Simulation processing
\end{abstract}

\begin{abstract}
Through the research of $\mathrm{CNC}$ lathe automatic programming and machining simulation parameters, summed up in the CNC lathe processing of complex shape programming procedure has advantages, and the complex parts processing as an example, the processing technology and NC program is complete, the use of CATIA V5 R20 plays an important role in the processing of parts. It is beneficial to the improvement of the machining process of the NC programmer, so as to solve the problems encountered in the numerical control machining. It is helpful for enterprises to save time and improve efficiency. The promotion of software automatic programming, so that more CNC practitioners to realize the importance of automatic programming software, and thus stimulate the interest of learning software automatic programming. The importance of automatic programming to the importance of automatic programming and the need for skilled use of automatic programming.
\end{abstract}

\section{Introduction}

In modern industry, the mechanization of production process, automation has become a prominent theme. Automation of continuous production process, such as chemical industry, has been basically solved. The special machine tool is an effective method for mass production automation, and it is an important way to control the automatic production of many varieties and small batch production. However, in addition to cutting itself, there are a large number of loading and unloading, handling, assembly and other operations, to be further mechanized, industrial manipulator is to achieve the automation of these processes. The mechanical hand is part of the function to imitate the human upper limb, it can automatically control it in accordance with the predetermined requirements of automated production equipment to transport products or tools to maintain the operation of the. Since the last century, in $60 \mathrm{~s}$, the PLC manipulator design is implemented as a product, development and application of it in the unceasing development, it can reduce the heavy manual labor, improve working conditions and safety production; improve production efficiency, stable product quality, reduce scrap rate, reduce production costs and enhance the competitiveness of enterprises plays its important role.[1]

\section{Process of Manipulator}

The structure of the manipulator and each part of the action diagram, as shown in the following figure. The manipulator are driven by a motor, its up and down, left and right are motor driven screw rod rotation to complete.

Analysis process [2]

The initial position of the manipulator stops at the origin, press the start button, the manipulator will fall to rise -- -- -- -- work right down to relax in the left parts -- up eight movements, to complete a work cycle. Drop, manipulator, right and left movements such as conversion, by the corresponding limit switch to control, and step up, relax action has time to control the conversion.

Control requirements 
In order to meet the requirements of production, the manipulator set up the manual operation and automatic operation mode, and automatic operation is divided into single step, single cycle and continuous operation mode.

Manual operation: the use of buttons to control every step of the manipulator. For example, press the "drop" button, the manipulator drops; press the "rise" button, the manipulator up. Manual operation can be used to adjust the position of the work and emergency parking manipulator back to the origin.[3]

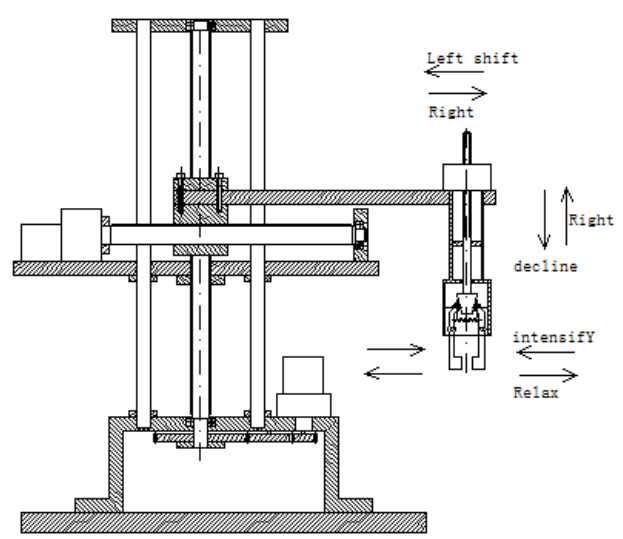

Figure 1. Schematic diagram of the manipulator structure and each part

\section{PLC Control System}

Determine the Input / Output Points and Select the Plc Model. input signal Position detection signal: the lower limit, the upper limit, the right limit, the left limit of a total of 4 travel switches, the need for 4 input terminals."No workpiece detection" signal: the use of photoelectric switch as a detection element, the need for 1 terminal. "Working mode" switch: there are 4 kinds of manual, single step, single cycle and continuous operation, the need for the 4 terminals such as transmission, Manual operation: the need for a decline, rising, right, left, up, loosen the 6 button, also need 6 input terminals, Automatic operation: still need to start, normal stop, emergency stop 3 buttons, also need the 3 input terminals. More than 18 input signals.[4] output signal, The PLC output is used to control the manipulator decline, rising, right, left, up, relax to three motor speed control, a total of 11 output points. Starting from the origin of the manipulator, the need for a light source, but also requires 1 output points. So, you need at least 6 output points. [5]Due to the control of the manipulator belongs to the switch control, there is no special requirement in function. Therefore, any type of small PLC can meet the requirements. According to the total number of points required for I/O and reserve a certain amount of spare, you can choose FX2N-48RM, its input and output of the 24 points, relay output. FX2N-48RM of the working parameters have been introduced in the second chapter, this is not in the introduction.[6]

Assign PLC Input / Output Terminals. PLC input and output terminals distribution diagram, as shown in fig. 2.

PLC Control System Programming. In order to facilitate programming, manual and automatic programs can be compiled separately relatively independent of the program segment, with the jump instruction selection, control system program structure diagram, as shown in fig. 5-3. Select the manual mode, X3 with the same, skip the automatic program, the implementation of manual procedures; choose the automatic mode of operation, X3 disconnected, the implementation of automatic procedures.[7]

Manual manual operation does not need to act in sequence, so it can be designed according to the general relay program. The ladder diagram of manual operation, as shown in Figure , the manual button X20-X25 control drop, rise, right, left, up and relax each action. In order to ensure the security of the system, some necessary chains are set up. In the left and right movement of the circuit to join the X11 as the upper limit of the chain, this is because the manipulator is only in the upper position, allowed to move left and right.[8] 


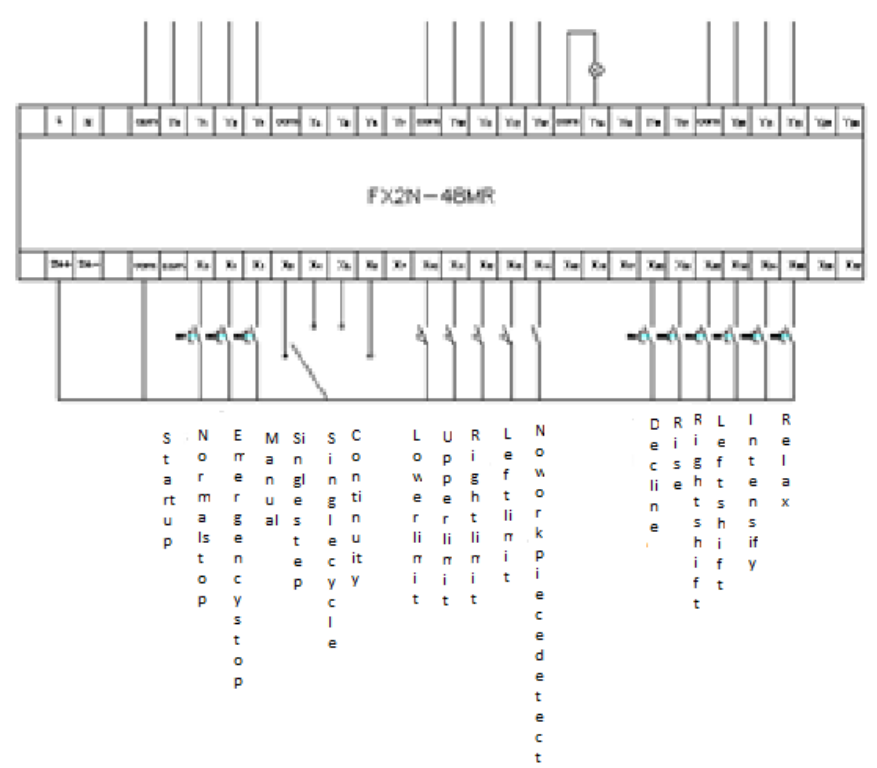

Figure 2. Input / output distribution diagram

The automatic program is shown in fig .

Continuous and single cycle operation. When the manipulator at the origin, the program is in the initial state of S0, the implementation of the decline. When the lower limit switch is turned on, the $\mathrm{X} 10$ is switched on, and the next state $\mathrm{S} 21$ is switched on, and then the next action is performed. When executing the last step, that is left to the origin of the encounter left limit switch, X13 switch, if it is a single cycle operation, M0 is disconnected, to return to the initial state, if the continuous operation, the M0 is switched on, state transfer to S20, and the beginning of the next cycle.[9]

In the operation, such as the normal stop button, then the X1 is connected, M0 reset, the manipulator's movement continues to perform a cycle, back to the initial state. If the emergency stop button is pressed, the X2 is switched on, the state S0 S33 is reset and the manipulator stops working. Restart, the first hand to move the manipulator back to the origin, in order to automatically operate again.

Single step operation. When the automatic program uses the step instruction design, the single step operation program is implemented with the "forbidden state transfer" marker M8040, as shown in Fig. 5-6. When the relay coil is switched on, the state of the stepping is prohibited, and the state transfer is allowed when the coil is powered off.

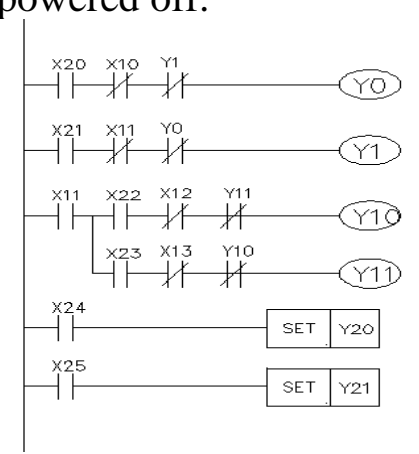

Figure 3. Manual procedures

In the single step operation mode, the start button X0 is used as a single step operation signal, and the X4 is connected. When the button is not pressed, the X0 is disconnected, the normally closed contact is closed, the M8040 is turned on, and the state transfer is prohibited.

When a step is completed, press the start button, the X0 is switched on, and the normally closed M8040 will be disconnected and the state will be transferred to the next step. 
The ladder diagram of the single step operation shown in Fig. 6 is connected to the upper end of the automatic program as shown in Fig. 5, and the whole ladder diagram, including single step。

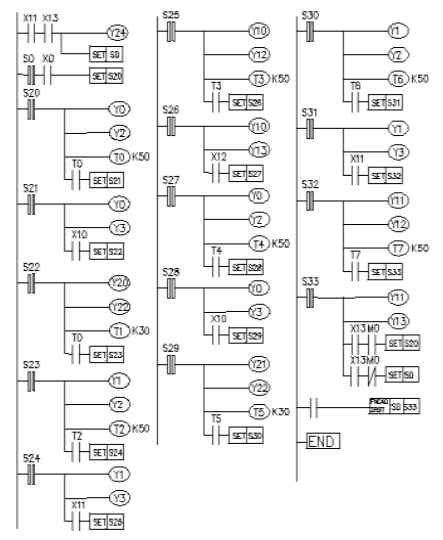

Figure 4. automatic program

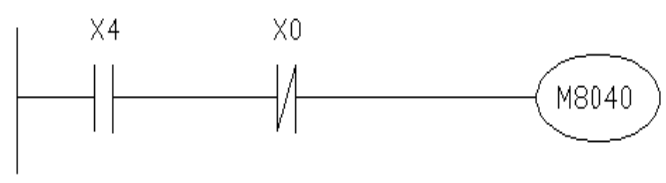

Figure 5. ladder diagram of a single step operation with "no state transfer"

At this point, the manipulator control program is completed. According to the block diagram of the main program shown in Fig. 4-3, the ladder diagram of the manual operation and the ladder diagram of the automatic program are embedded.

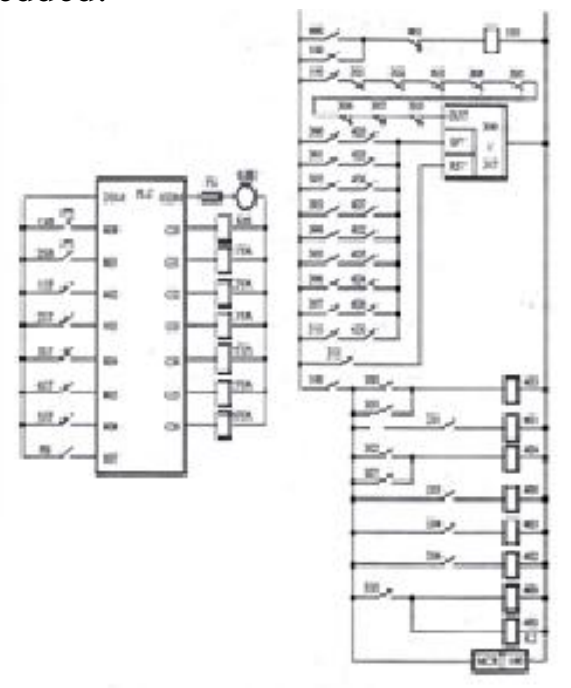

Figure 6. Ladder diagram of the whole program

The pulse shift register is shifted to the right one, "1" state, a corresponding auxiliary relay is set to "1", the normally open contacts, to complete the next action, and "STF" is give a shift pulse ready. When the shift register of the "1" state moves to 310 when you enter "relax" step 450, start time, time to $2 \mathrm{~S}$, normally open contacts 450 , "STF" end give pulse to make the shift register in the "1" state from 310 to 311311 of the normally open contacts to reset the shift register. At this time $301 \sim$ 310 of the normally closed contacts are closed, and the 300 is set to "1" program, the cycle begins anew from the first step.[10]

Stop, press the stop button $2 \mathrm{SB}, 401$ switch on, 401 of the normally closed contact is disconnected, so that the 100 off, the action stopped.

\section{Concluding Remarks}

Through the design of mechanical hand, understand the modern enterprise in the completion of automatic control can not be separated from the PLC control, it can make the enterprise at the same time reduce labor and production costs. At present, many enterprises in our country are still relatively backward in production equipment and most of the processes are completed by hand, and the cost is low and the operation is simple. But I believe that with the development of science and 
technology of multifunctional complex manipulator will appear in the near future, so we have not in the future study and life, learning and innovation, the only way to design more in line with the needs of modern social products.

\section{Reference}

[1] Xudong Deng: Research and development of electrical control system for distributed control system [J], Hubei electric power system, Vol. 25 No.5, p.21.

[2] Shenghua Feng: A discussion on the development of electrical control of elevator [J]. science and technology information, Vol. 21 (2009)

[3] Liling Liu: The development technology of electrical control technology [J]. Journal of Beijing Electric Power College, (2011) No.3, p.31.

[4] Guowei Zhu and Guoxiu Wang: Graphic LCD Module MGLS-19264. Application of Intelligent geophone [J]. Beijing Polytechnic College, (2003:2)

[5] Changchu Liao: PLC programming and application (Mechanical Industry Press, China 2014)

[6] Kewang Pang and Wenhua Yuan: PLC electrical control system design and application (China Electric Power Press, China 2014)

[7] Zhiyong Jia, Xueyong Li and Gao Hongyan: introduction to the application of PLC (China Electric Power Press, China 2014)

[8] Huafeng Mei: electrical equipment and control (China Water Conservancy and Hydropower Press, China 2015)

[9] Pin Wan and Dejie Lin: Electrical Testing Technology (Mechanical Industry Press, China 2015)

[10] Songwei Huang and Jinhui Zou: electrical control and PLC application technology (Electronic Industry Press, China 2015) 